\title{
Design of OFDM-CPM Transceiver and Its Application in Optical Access System
}

Yufeng Shao*, Anle Fang, Liandong Wang, Jiancun Zuo, Jianjun Wang, Qiudong Sun, Hongxuan Xu

Faculty of Engineering, Shanghai Second Polytechnic University,

Shanghai, China, 201209

*syufeng@163.com

\begin{abstract}
.
A novel OFDM transceiver is designed and demonstrated in this work for optical access uplink and downlink transmission applications using continuous phase modulation (CPM) and demodulation. Electronic OFDM-CPM signals are offline generated by an arbitrary waveform generator (AWG) and a single-arm Mach-Zehnder modulator is used to generate optical OFDM-CPM signals. The result show the transmission and reception performance of optical signals with OFDM-CPM transceiver after transmission over SMF-28 is good.
\end{abstract}

Keywords: Optical access system; OFDM-CPM; Transceiver.

\section{Introduction}

Recently, are many research groups have demonstrated optical orthogonal frequency division multiplexing (OFDM) signals can be widely applied in high speed optical transmission system and flexible configuration optical access networks [1-4]. Adopting optical OFDM signals, not only high spectral efficiency (SE) can be achieved, but also we can obtain good performance against chromatic dispersion (CD) and polarization mode dispersion (PMD) using digital signal processing (DSP). Currently, the OFDM has been regarded as an excellent candidate to be used in optical access system. The reason is the optical fiber is been widely used as the uplink and downlink transmission media and OFDM optical access system can support different communications services. Hence, the design of OFDM transceiver has attracted much attention in both industry and 
academia. For example, researchers are working on the OFDM development of next generation passive optical network (PON) and radio over fiber system (RoF). Moreover, upgradeability, and cost-effective configuration, and the design of optical OFDM access system architecture is expected to be compatible with commercial access system without any change of optical line terminal (OLT) and optical networking unit (ONU) configuration to serve various users, which is shown in Fig.1 [5-7]. Especially, OFDM technique is been adopted in optical access system has other advantages compared to common technologies: (1) low bandwidth can support high data rate while high modulation; (2) flexibility in adjusting bandwidth resource sharing and virtualization; subsets of sub-carriers can support digital and analog signals with different quality of services requirements; (3) it is a scalable configuration can coexist with time division multiplexing (TDM) and wavelength division multiplexing (WDM) techniques. As we know, as a high spectral-efficiency modulation format, OFDM signals are generally generated by MQAM or MPSK coding, which possibly causes discontinuous phase transition between the adjacent symbols after transmission. Hence, the digital signal processing (DSP) at receiver is complex and unstable. The binary minimum shift keying (MSK) is one special form of continuous phase modulation (CPM), has the property of decaying fast side-lobe compared with other modulation. While using CPM as the coder for OFDM generation, we can mitigate the effect of phase noise and carrier frequency offset, and thus reduces the inter-carrier interference (ICI) that caused by other reasons. In this work, we experimentally demonstrate novel subcarrier OFDM-CPM bidirectional transceivers in the full-duplex optical access system. In the above system, of 


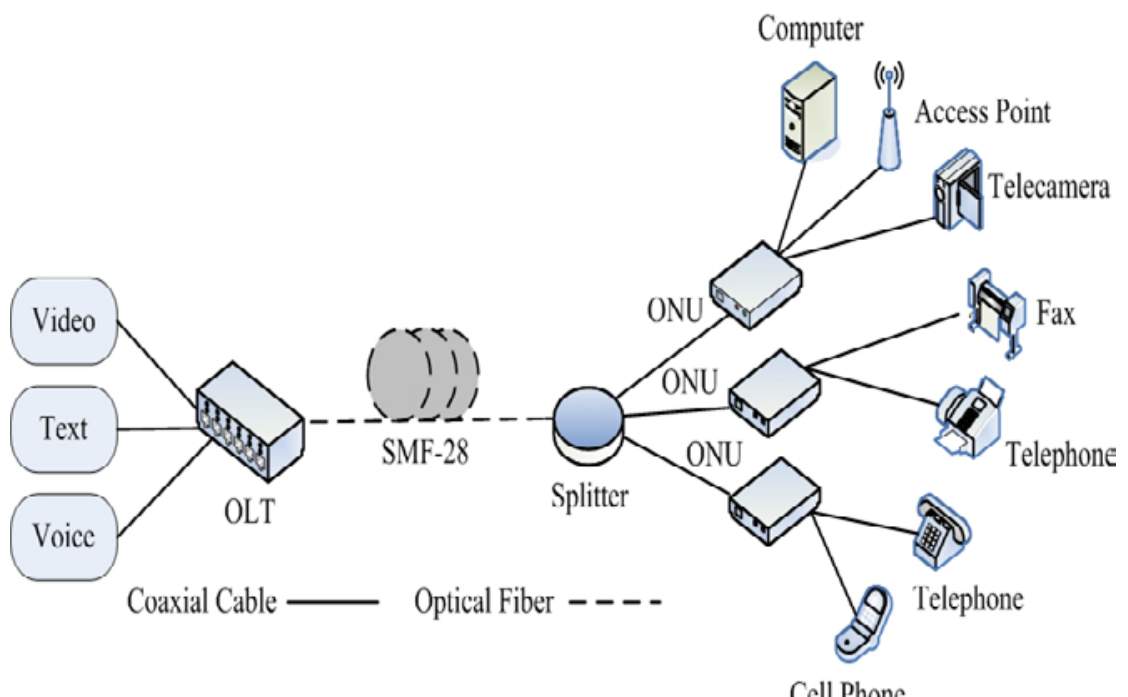

Figure 1. Optical access system. OLT: optical line terminal; SMF: single mode fiber; ONU: optical network unit.

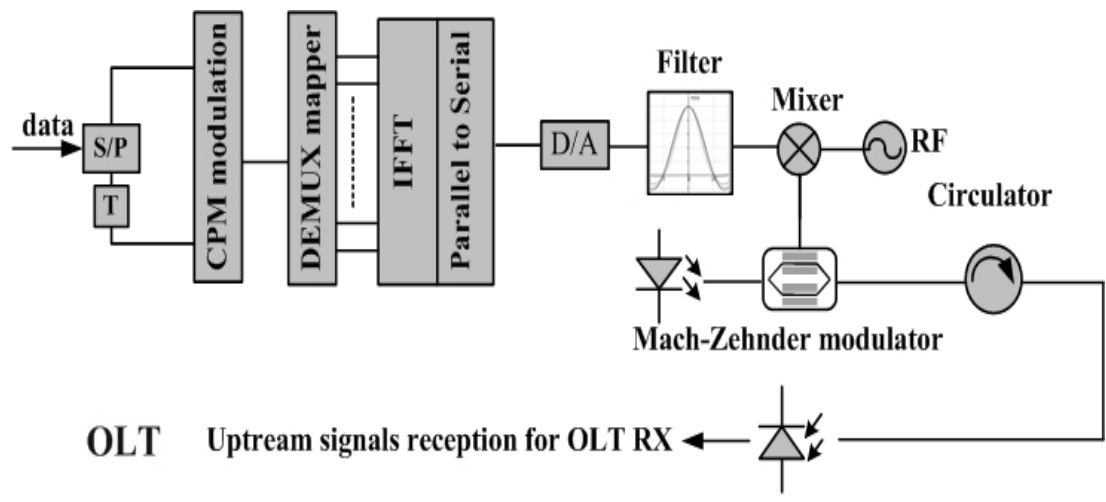

Figure 2. Configuration of transceiver at the OLT.

OFDM-CPM subcarrier downstream signals, the central carrier will be filtered out through using one optical band pass filter (OBPF) and re-modulated with upstream OFDM-CPM signals in the ONU. Experimental results show it is a cost-effective solution scheme in the future optical access system as well as its flexible and convenient capacity upgrading.

\section{System Configuration and Analysis}


Fig.2 shows the configuration of transceiver at the OLT. One continuous-wave (CW) beam is generated by a distribute feedback (DFB) laser at frequencies mapped 193.1THz. One input data is first serial parallel transformed and I/Q in the CPM modulation part. CPM is used to map bit stream data to the OFDM sub-carriers, and there are 1024 sub-carriers. The OFDM-CPM baseband waveforms produced by the arbitrary waveform generator (Tektronix AWG710) are continuously output at the sample rate of $20 \mathrm{GHz}$ (8-bits digital-to-analog converter). The used guard interval is equal to one quarter of the observation period, and the fixed cyclic prefix (CP) is applied. The 2.5Gb/s OFDM-CPM baseband signals are generated offline and then up-converted to $20 \mathrm{GHz}$ to realize subcarrier OFDM-CPM signals by an electrical mixer. At the OLT terminal, the generated subcarrier OFDM-CPM signal is used to directly modulate a $\mathrm{LiNbO}_{3}$ Mach-Zehnder Modulator. The generated one optical OFDM-CPM signal is filtered by an optical filter, and the bandwidth of the filter is set to $10 \mathrm{GHz}$.

We apply commonly used fiber parameters in this work: fiber CD of $16.1 \mathrm{ps} / \mathrm{nm} / \mathrm{km}, 0.2 \mathrm{~dB} / \mathrm{km}$ loss, and a nonlinear coefficient of $2.6 \times 10^{-20} \mathrm{~m}^{2} / \mathrm{W}$, as shown in TABLE I. After transmission over 20-km standard single-mode fiber (SMF-28), the generated 50\% RZ-8PSK optical signals from the OLT

TABLE I. Downlink parameters

\begin{tabular}{cc}
\hline Fiber parameters & SMF-28 \\
Length $(\mathrm{km})$ & 25 \\
Attenuation $(\mathrm{dB} / \mathrm{km})$ & 0.2 \\
Dispersion $(\mathrm{ps} / \mathrm{nm} / \mathrm{km})$ & 16 \\
Dispersion slop $\left(\left(\mathrm{ps} / \mathrm{nm}^{2} / \mathrm{km}\right)\right.$ & 0.08 \\
Nonlinear coefficient $\left(\mathrm{W}^{-1} \mathrm{~km}^{-1}\right)$ & 2.6 \\
Core area $\left(\mu \mathrm{m}^{2}\right)$ & 84.9 \\
\hline
\end{tabular}




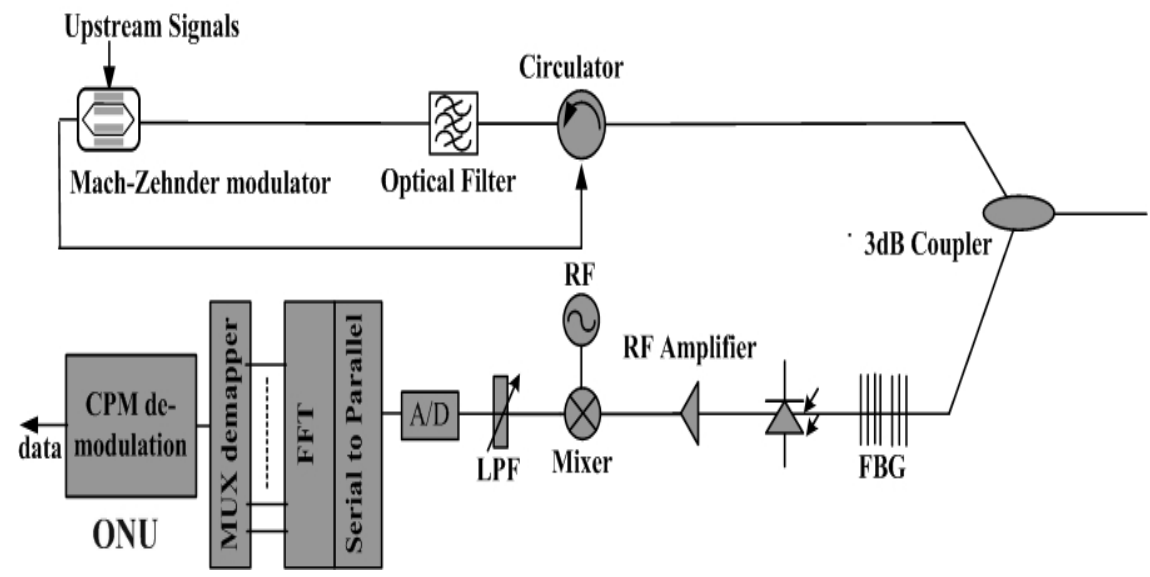

Figure 3. Configuration of transceiver at the ONU.

terminal is amplified through an erbium-doped fiber amplifier (EDFA) for compensating the downlink attenuation and split into one ONU part.

After transmission over 25km standard single-mode fiber (SMF-28), OFDM-CPM signals from the OLT are split into different ONU parts. As shown in Fig.3, the adopted ONU terminal has two parts, one is the downstream reception part and the other is OFDM-CPM upstream signals re-modulation part. In the downstream reception part, the generated $40 \mathrm{GHz}$ subcarrier carrying 2.5Gb/s OFDM-CPM downstream signal is filtered by one fiber Bragg grating (FBG) and then detected by O/E conversion via a PIN PD with a 3-dB bandwidth of $60 \mathrm{GHz}$. The converted electrical OFDM-CPM signal is amplified by an RF amplifier with a bandwidth of $2.5 \mathrm{GHz}$ centered at $40 \mathrm{GHz}$. An electrical local oscillator (LO) signal at $40 \mathrm{GHz}$ was generated and we adopted it and a mixer to down-convert the electrical mm-wave OFDM-CPM signal. The down-converted 2.5Gb/s OFDM-CPM downstream signal is detected after passing low passing filter (LPF). In OFDM-CPM upstream signals re-modulation part, the central carrier of the received optical OFDM-CPM signal frequency is filtered out through one optical filter and then used to be re-modulated by upstream signals.

\section{Results}


Fig.4 and Fig.5 show the corresponding constellation diagrams after downlink and uplink transmission. It is clear to see, the constellation track curves show the OFDM-CPM signal has the property of phase continuity and this feature will reduce the complexity of transceiver design and DSP at the OLT and the ONU. Furthermore, the constellation track curves after uplink transmission are worse than after downlink transmission because the receiver sensitivity degradation of uplink OFDM-CPM signals.

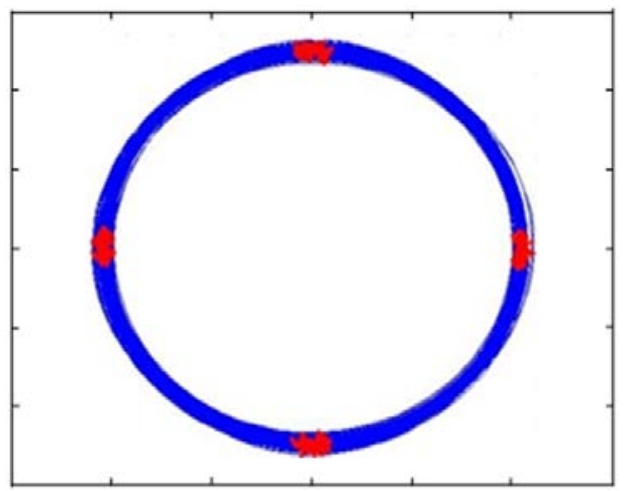

Figure 2. Not using phase equalization in MSPE

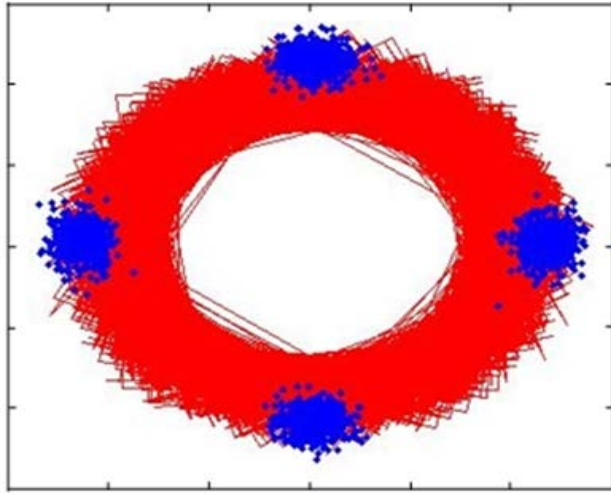

Figure 3. Using phase equalization in MSPE.

\section{Summary}

We have proposed and experimentally demonstrated a novel scheme to set up a bidirectional transceivers system with $2.5 \mathrm{~Gb} / \mathrm{s}$ OFDM-CPM signals. For OFDM-CPM signals bidirectional transmission, slighter downstream 
transmission impairments are introduced than upstream signals, while the signal passes through 25km SMF-28. Because CPM is a special type of binary continuous phase-frequency shift keying, and the generation and reception of optical OFDM-CPM signals as bidirectional transmission signals can simplify digital signal processing compared with OFDM-QPSK, and it is a competitive scheme in optical access system.

\section{Acknowledgment}

This work is partially supported by the National Natural Science Foundation of China (No. 61107064), Leading Academic Discipline Project of Communication and Information System (No. XXKZD1302), University Young Teachers cultivation Plan of Shanghai (No. ZZegd14003), and School Foundation of Shanghai Second Polytechnic University (No.EGD14XQD01).

\section{References}

[1] Y. Shao, S. Wang, and Z. Tan, et al. Seamless Integration of RZ-DQPSK-DWDM Optical Links with MISO-OFDM-QPSK System for 4th Generation Wide-Area Coverage Mobile Communication. Microwave and Optical Technology Letters, 2014, vol.56, Page(s): 797-801.

[2] Y. Shao, Y. Wang, N. Chi. 60-GHz RoF System with Low PAPR 16QAM-OFDM Downlink Using PTS Segmentation. IEEE Photonics Technology Letters, 2013, vol.25, Page(s): 855-858.

[3] Y. Shao, N. Chi, and J. Fan, et al. Generation of 16-QAM-OFDM signals using selected mapping method and its application in optical millimeter-wave access system. IEEE Photonics Technology Letters, 2012, vol.24, Page(s): 1301-1303.

[4] Y. Shao, N. Chi. A Novel Scheme for Seamless Integration of RZ-DPSK-DWDM Optical Links with MIMO-OFDM System. Microwave and Optical Technology Letters, 2012, vol.54, Page(s): 1676-1679. 
[5] D. Qian, N. Cvijetic, J. Hu et al. Optical OFDM transmission in metro/access Networks. OFC, 2009, Paper OMV1.

[6] C. W. Chow, C. H. Yeh, C. H. Wang. Demonstration of high spectral efficient OFDM-QAM long reach passive optical networks. ECOC, 2008, Paper Th.2.F.5.

[7] P.L.Tien, Y.M.Lin, M.C.Yuang. A novel OFDMA-PON architecture toward seamless broadband and wireless Integration. OFC, 2009, Paper OMV2. 